Z Gerontol Geriat 2009 · 42:423-424 DOI 10.1007/s00391-009-0069-x

Online publiziert: 28. Oktober 2009

(c) Springer-Verlag 2009

\author{
G. Naegele ${ }^{1} \cdot D$. Schaeffer ${ }^{2}$ \\ ${ }^{1}$ Institut für Gerontologie an der Technischen Universität Dortmund \\ ${ }^{2}$ Fakultät für Gesundheitswissenschaften, Universität Bielefeld
}

\title{
Gesundheitspolitik in einer alternden Gesellschaft
}

Die Anpassung der gesundheitlichen Versorgungssysteme an eine schnell alternde Gesellschaft steht schon seit Längerem als Forderung im Raum. Erinnert sei in diesem Zusammenhang nur an eine frühere Station der Debatte.

So hat schon die Bundestags-EnquêteKommission „Demographischer Wandel“ in ihrem Abschlussbericht von 2002 wie folgt argumentiert:

„Die durch die demographische Entwicklung sich ergebende veränderte Bedarfslage gesundheitlicher Versorgung älterer Menschen steht einem Versorgungssystem gegenüber, das in der Praxis den veränderten Anforderungen nur unzureichend gerecht wird.“ „Die quantitative Zunahme der älteren Menschen und vor allem der hochaltrigen mit ihrem höheren Behandlungs- und Pflegebedarf (...) muss noch stärker zum bestimmenden Faktor bei der Entwicklung des Gesundheitswesens werden." ([3], S. 390)

Vergleichbar war auch der Tenor des 3. und 4. Altenberichts $[1,2]$ und der Bericht der NRW-Enquête-Kommission „Situation und Zukunft der Pflege NRW“ [6]. Seither sind weitere Jahre ins Land gegangen. Doch kann man nicht gerade behaupten, dass sich die Probleme entschärft hätten und flächendeckend die richtigen gesundheitspolitischen Wege beschritten seien. Ein Problem ist zudem, dass die gegenwärtige Diskussion zur Zukunft der gesundheitlichen Versorgung älterer Menschen primär unter finanzierungspolitischem Vorzeichen geführt wird und weniger unter dem Blickwinkel einer „fachlich angemessenen, bedarfs- und bedürfnisgerechten Versorgung" ([4], S. 9). Noch immer dominiert in Deutschland die kurative Medizin, ste- hen chronische Erkrankungen und Multimorbidität nicht im Zentrum der medizinischen Versorgung, spielt die Geriatrie innerhalb der etablierten Medizin (einschließlich der Ausbildung) nur eine untergeordnete Rolle, werden in der vorherrschenden Gesundheitspolitik die besonderen Krankheitsrisiken älterer, v. a. hochaltriger Menschen nicht als komplexe Bedarfslagen auch jenseits der Medizin konzeptualisiert, findet kaum Koordination und Kooperation zwischen den verschiedenen, an der gesundheitlichen Versorgung älterer Menschen beteiligten medizinischen, pflegerischen, psychosozialen und hauswirtschaftlichen Diensten und Gesundheitsprofessionen statt, sind Gesundheitsförderung, Prävention und Rehabilitation für bzw. bei alten Menschen unterentwickelt, gelten Pflege und Palliativversorgung nicht als gleichberechtigte Teile in einem Gesamtversorgungssystem etc.

Das vorliegende Heft thematisiert derartige Versorgungserfordernisse und -optionen aus verschiedenen Blickwinkeln. Der Zeitpunkt dafür könnte günstiger nicht sein. Soeben hat der Sachverständigenrat zur Begutachtung der Entwicklung im Gesundheitswesen (SVR) ein Sondergutachten zum Thema „Koordination und Integration - Gesundheitsversorgung in einer Gesellschaft des langen Lebens" vorgelegt [8]. Nahezu zeitgleich hat eine Expertengruppe im Auftrag der Friedrich-Ebert-Stiftung ein Positionspapier zum Thema „Zukunft des Gesundheitssystems, solidarisch finanzierte Versorgungssysteme für eine alternde Gesellschaft" erarbeitet und publiziert [4]. Beide kommen zu ähnlichen Ergebnissen, bei- de finden in diesem Heft ein zusätzliches Forum.

Es ist unser Wunsch, mit diesem Heft dazu beizutragen, auch die gerontologisch-geriatrische Diskussion bzw. „scientific community" stärker zu sensibilisieren. Wir erhoffen uns damit auch von dieser Seite Unterstützung für das Anliegen, die gesundheitliche Versorgung in einer alternden Gesellschaft nicht etwa als „finanzielle Zeitbombe“ zu sehen und politisch zu behandeln, sondern sie als eine fachliche und zugleich multidisziplinäre Gestaltungsaufgabe zu begreifen, die sich primär an dem Bedarf und den Bedürfnissen älterer und insbesondere hochaltriger Menschen ausrichtet.

Zugleich wollen wir explizit auch auf sozial bedingte Ungleichheiten im Krankheitsrisiko auch älterer Menschen und auf deren häufig lebenslange Vorprägung hinweisen. So lassen vorliegende Befunde erkennen, dass ein Zusammenhang zwischen sozioökonomischem und gesundheitlichem Status auch im höheren Alter zu beobachten ist, wobei insbesondere dem Einkommen als Prädiktor der Morbidität und Mortalität Bedeutung zugeschrieben wird. Gesundheit und Krankheit lassen sich zu einem guten Teil als Folge einer Kumulation von günstigen Entwicklungen wie auch von Risiken und Benachteiligungen beschreiben. Unter Berücksichtigung der Lebenslaufperspektive ist dabei festzuhalten, dass sich im gesamten Lebensverlauf - nicht nur in den ersten Lebensphasen - gesundheitsschädigende Faktoren etablieren und das Risiko für chronische Krankheiten und Sterblichkeit erhöhen können. Tendenziell ist zu beobachten, dass sich (Gesundheits-)Risiken von einer Lebensphase zur nächsten auf- 
schichten und so in ihrer Bedeutung zunehmen. In der geriatrischen wie auch insgesamt in der (sozial)medizinischen Forschung in Deutschland sind derartige lebenslaufbezogenen Zusammenhänge allerdings bislang zu wenig untersucht $[5,7]$.

Der Beitrag von A. Kuhlmey basiert auf dem 2009 erschienenen Sondergutachten des SVR, dessen Mitglied sie seit vielen Jahren ist. Er befasst sich mit den besonderen Anforderungen an die Versorgung alter Menschen, die - wie ausgeführt wird - v. a. auf Phänomene wie chronische Krankheit, Multimorbidität, Polypharmazie und Pflegebedürftigkeit zurückgehen. Diskutiert wird außerdem, welche gesundheits- und versorgungspolitischen Schritte erforderlich sind, um diese Anforderungen zu bewältigen. Neben dem Ausbau von Prävention gehören dazu die qualitative Weiterentwicklung der ambulanten Versorgung und Pflege unter Einbeziehung regionaler Spezifika, der Ausbau von Case- und Care-Management, die Modernisierung der Langzeitund Heimversorgung sowie der Ausbau der Palliativversorgung.

G. Naegele stellt in seinem Beitrag wichtige Ergebnisse des oben erwähnten Gutachtens der Friedrich-Ebert-Stiftung vor. Dabei geht es v. a. um Perspektiven einer bedarfs- und bedürfnisgerechten gesundheitlichen Versorgung für ältere Menschen vor dem Hintergrund des demografischen Wandels. „In einer schnell alternden Gesellschaft muss es (demnach: G.N. und D.S.) prioritäres Ziel der Gesundheitspolitik sein, die bestehenden gesundheitlichen Versorgungssysteme bedarfs- und bedürfnisgerecht an die sich ändernden Anforderungen auszurichten und insbesondere ihre jeweiligen Handlungslogiken auf die Besonderheiten geriatrischer Krankheitsbilder anzupassen."

D. Schaeffer und A. Büscher setzen sich mit Fragen der Prävention und Gesundheitsförderung auseinander und widmen sich dabei einem bislang kaum in den Blick genommenen Setting: der Langzeitversorgung und ihren meist hochbetagten Nutzern. Sie knüpfen an ein gemeinsam mit dem Ludwig Boltzmann Institut, Wien, durchgeführtes Projekt zur Gesundheitsförderungsforschung an und diskutieren, was Prävention und Ge- sundheitsförderung angesichts der aktuellen Problemsituation der Langzeitversorgung in Deutschland, aber auch angesichts der besonderen Vulnerabilität der Nutzer bedeuten können und wie sie zu konzeptualisieren sind.

M. Reichert berichtet über Ergebnisse des 2008 abgeschlossenen EU-Projektes „HealthProElderly“, das zum Ziel hatte, in 11 EU-Ländern Kriterien zu identifizieren, die den Erfolg von Gesundheitsförderungsprogrammen für ältere Menschen nachhaltig gewährleisten. Ausgehend von einer Literaturanalyse zur Ermittlung des Status quo der Gesundheitsförderung in den beteiligten Ländern und einer Evaluierung von innovativen „Best-practiceProjekten“ werden Kriterien für eine erfolgreiche Gesundheitsförderung im Alter entwickelt. Dazu gehören u. a. Zielgruppenbezug, Settingansatz und Ganzheitlichkeit. Der Beitrag endet mit Leitlinien und Empfehlungen für Politik und Praxis und fragt dabei auch nach ihrer Übertragbarkeit auf Deutschland.

O. von dem Knesebeck und N. Vonneilich wenden sich dem Thema gesundheitliche Ungleichheit im Alter zu und geben einen Überblick über die vorliegenden Studien und empirischen Befunde. Dabei präsentieren sie auch eigene Studienergebnisse zum Zusammenhang von Bildung und Depression im höheren Lebensalter. Ebenso diskutieren sie unter Rückgriff auf die Lebenslaufperspektive mögliche Gründe für soziale und gesundheitliche Ungleichheit im Alter und leiten daraus abschließend gesundheitspolitische Empfehlungen ab.

Dortmund und Bielefeld, im September 2009

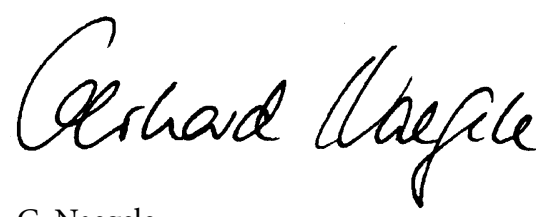

G. Naegele
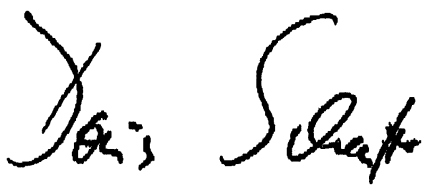

D. Schaeffer

\section{Korrespondenzadresse}

Prof. Dr. G. Naegele

Institut für Gerontologie

an der Technischen Universität Dortmund,

Evinger Platz 13, 44339 Dortmund

orka@post.uni-dortmund.de

\section{Literatur}

1. BMSFJ (2002) Risiken, Lebensqualität und Versorgung Hochaltriger - unter besonderer Berücksichtigung demenzieller Erkrankungen, Vierter Bericht zur Lage der älteren Generation in der Bundesrepublik Deutschland. BMSFJ, Berlin

2. BMFSFJ (2001) Alter und Gesellschaft, Dritter Bericht zur Lage der älteren Generation in der Bundesrepublik Deutschland. BMSFJ, Berlin

3. Deutscher Bundestag (2002) Schlussbericht der Enquête-Kommission „Demographischer Wandel Herausforderungen unserer älter werdenden Gesellschaft an den Einzelnen und die Politik". Drucksache $14 / 880014$. Wahlperiode

4. FES - Friedrich-Ebert-Stiftung (2009) Zukunft des Gesundheitssystems. Solidarisch finanzierte Versorgungssysteme für eine alternde Gesellschaft, WISO Diskurs, Juni 2009. FES-Eigenverlag, Berlin

5. Hurrelmann K, Schaeffer D (2006) Lebenslauf und Gesundheit. Public Health Forum 14(50):4-6

6. Landtag NRW - Enquête-Kommission (2005) Situation und Zukunft der Pflege in NRW, Bericht der Enquête-Kommission des Landtags von Nordrhein-Westfalen, Düsseldorf

7. Schaeffer D (2009) Bewältigung chronischer Krankheit im Lebenslauf. Huber, Bern

8. SVR - Sachverständigenrat zur Begutachtung der Entwicklung im Gesundheitswesen (2009) Koordination und Integration - Gesundheitsversorgung in einer Gesellschaft des längeren Lebens, Sondergutachten 2009. Verfügbar unter: http:// www.svr-gesundheit.de/ Startseite/Startseite.htm (14.07.2009) 